\title{
Synthesis and Structure Elucidation of New Benzimidazole Amidoxime Derivatives
}

\section{Yeni Benzimidazol Amidoksim Türevlerinin Sentezleri ve Yapı Aydınlatmaları}

\author{
(1) Cigdem KARAASLAN* \\ Ankara University Faculty of Pharmacy, Department of Pharmaceutical Chemistry, Ankara, Turkey
}

\begin{abstract}
Objectives: In our previous studies we synthesized some potent antiparasitic, anticancer and antimicrobial amidine derivatives. Despite all their potent activities, it is well known that due to their cationic charge, amidine derivatives pose a serious problem in terms of bioavailability. The main purpose of this study is to prepare amidoxime derivatives of previously synthesized potent amidine derivatives as prodrugs in order to increase their bioavailabilities.

Materials and Methods: The targeted benzimidazole amidoximes were synthesized from their nitrile derivatives. The nitrile groups of these benzimidazole carbonitriles were converted to $\mathrm{N}$-hydroxy benzamidine derivatives (amidoxime derivatives, 20-29) in the presence of $\mathrm{NH} \mathrm{H}_{2} \mathrm{OH}$. $\mathrm{HCl}$ and $\mathrm{KO}-\mathrm{t}-\mathrm{Bu}$ in dimethyl sulfoxide. Structures of newly synthesized amidoxime derivatives were elucidated with $1 \mathrm{H}-\mathrm{NMR}$, $13 \mathrm{C}-\mathrm{NMR}$ and some $2 \mathrm{D}$ NMR techniques like COSY, NOESY, HSQC and HMBC.

Results: A new series of benzimidazole amidoximes were synthesized and their structural elucidations were done in this study.

Conclusion: In order to solve the potential bioavailability problem of potent amidine derivatives, we prepared the prodrugs of those potent amidine derivatives as their amidoxime derivatives. In vivo studies of both previous amidine derivatives and amidoxime prodrugs of those amidines which were synthesized in this study are planned to perform in our ongoing studies.
\end{abstract}

Key words: Amidoxim, amidinobenzimidazole, prodrug, 1H-NMR, 2DNMR

öz

Amaç: Daha önce yaptığımız çalışmalarda, antiparaziter, antikanser ve antimikrobiyal etkili potent aktiviteye sahip bazı amidin türevleri sentezledik. Ancak potent aktivitelerine rağmen, amidin türevlerinin, katyonik yükleri nedeniyle biyoyararlanım açısından ciddi bir sorun oluşturduğu bilinmektedir. Bu çalıșmanın temel amacı, daha önce sentezlenen etkili amidin türevlerinin biyoyararlanımlarını artırmak için, ön ilaçları olarak bilinen amidoksim türevlerini hazırlamaktır.

Gereç ve Yöntemler: Hedeflenen benzimidazol amidoksimler, nitril türevlerinden hareketle sentezlenmiştir. Bu benzimidazol karbonitrillerin nitril grupları, dimetil sülfoksit içerisinde $\mathrm{NH}_{2} \mathrm{OH} . \mathrm{HCl}$ ve $\mathrm{KO}$-t-Bu varlığında N-hidroksi benzamidin türevlerine (amidoksim türevleri, 20-29) dönüștürülmüştür. Yeni sentezlenmiş olan amidoksim türevlerinin yapıları ise 1H-NMR, 13C-NMR ve COSY, NOESY, HSQC ve HMBC gibi bazı 2D NMR teknikleri ile açıklanmıştır.

Bulgular: Bu çalışmada yeni bir seri benzimidazol amidoxim türevi bileşik sentezlenmiş ve yapıları aydınlatılmıştır.

Sonuç: Daha önceki çalışmalarımızda sentezlediğimiz amidin türevleri ile literatürlerde biyolojik açıdan etkili bulduğumuz amidin türevlerinin potansiyel biyoyararlanım problemlerini çözebilmek için, bu çalışmada söz konusu potent amidinlerin ön ilaç formları olan amidoksim türevlerini hazırladık. Etkili amidin türevleri ile bu çalışmada sentezlenen amidoksim türevlerinin in vivo çalışmalarının devam eden araștırmalarımız kapsamında yapılması planlanmaktadır.

Anahtar kelimeler: Amidoksim, amidinobenzimidazol, önilaç, 1H-NMR, 2DNMR

*Correspondence: E-mail: karaslan@pharmacy.ankara.edu.tr, Phone: +90 3122033063 ORCID-ID: orcid.org/0000-0002-2006-5421

Received: 04.12.2019, Accepted: 16.12.2019

-Turk J Pharm Sci, Published by Galenos Publishing House. 


\section{INTRODUCTION}

A biologically active compound with intended pharmacological activity may have unwanted properties that limit its bioavailability or structure which negatively effect its activities in the organism. Amidoximes are generally developed to overcome low oral bioavailability of amidines which are pharmacologically effective in many areas including antiparasitic, ${ }^{1}$ antimicrobial ${ }^{2}$ and anticancer activities. ${ }^{3}$

Amidine derivatives which are known as DNA interactive compounds, have been used in clinic for many years especially against protozoal diseases. The most important example of this group is pentamidine (Figure 1) which has been used effectively in the treatment of several protozoal diseases for many years. ${ }^{1.4}$ Amidino group bearing compounds with similar structures such as berenil, furamidine (Figure 1) and some amidino benzimidazoles are also used as effective antiprotozoal compounds based on their selective binding to AT-rich sequences of DNA. ${ }^{5}$ Furthermore, it is also known that these compounds have shown very good activities in anticancer therapy. ${ }^{6-8}$ Pentamidine has been emphasized as a potential anticancer agent also. ${ }^{9-11}$ Although amidine group is essential for the pharmacological effect of several active compounds, their oral bioavailability is too low and they have several toxic effects. Due to hydrophilic and very strong basic properties of amidines, after protonation they form highly mesomerically stabilized cations and so they are usually incapable of passing through membranes and cannot be absorbed from the gastrointestinal system after oral administration. ${ }^{12}$ In order to avoid this problem, several prodrug attempts have been performed on the amidine moiety of drugs and hydroxylation of amidine group to amidoxime has been found the most promising alternative.
Amidoximes instead of amidines principle was first performed to pentamidine and then it has been transferred to several other amidine derivatives (Furamidin-Pafuramidin) (Figure 1) for increasing oral absorption and improving bioavailability. ${ }^{12-14}$

Amidoxime derivatives indicate a prodrug class used to enhance the oral bioavailability of amidine containing drugs. Because of their lower basicity and higher lipophilicity than amidine derivatives, they can be quickly absorbed by the gastrointestinal tract after oral administration. ${ }^{12,15}$

Over the past decade, we have focused our effort on the design of amidino benzimidazole derivatives possessing antiprotozoal and anticancer activity.,3

Mono-di amidino 2-anilino benzimidazoles were designed, synthesized and their antiprotozoal activities were determined against Trypanosoma brucei rhodesiense and Plasmodium falciparum. In this study some of dicationic compounds (Figure 2a) showed almost equal activity with melarsoprol against Trypanosoma brucei rhodesiense and they showed close activity with chloroquine against Plasmodium falciparum!

Furthermore, the anticancer activities of these compounds with additional new analogues were studied against MCF-7 human breast adenocarcinoma cells. Some of them (Figure 2b) strongly inhibited MCF-7 cell viability compared to clinically used reference compounds, docetaxel and imatinib mesylate. ${ }^{3}$

Recently we reported synthesis and antimicrobial-anticancer activities of 2-(3,4-dimethoxyphenyl) benzazoles and imidazopyridine derivatives with very important results ${ }^{16}$ some of which bearing amidine groups (Figure 2c).

As a part of our continuing research program, focused on developing new antimicrobial and anticancer benzimidazole

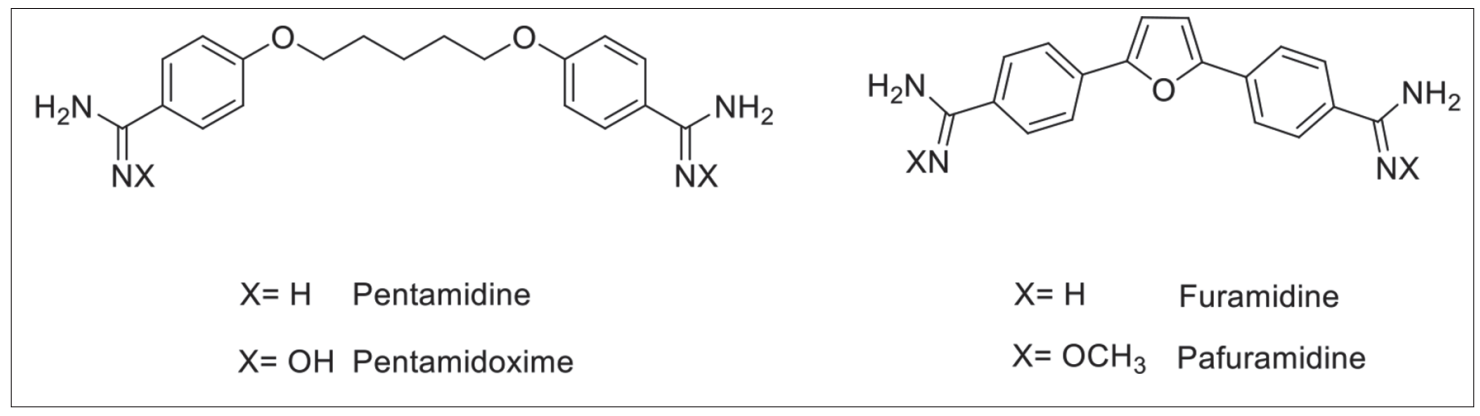

Figure 1. Chemical structures of some amidine derivatives and their amidoxime prodrugs

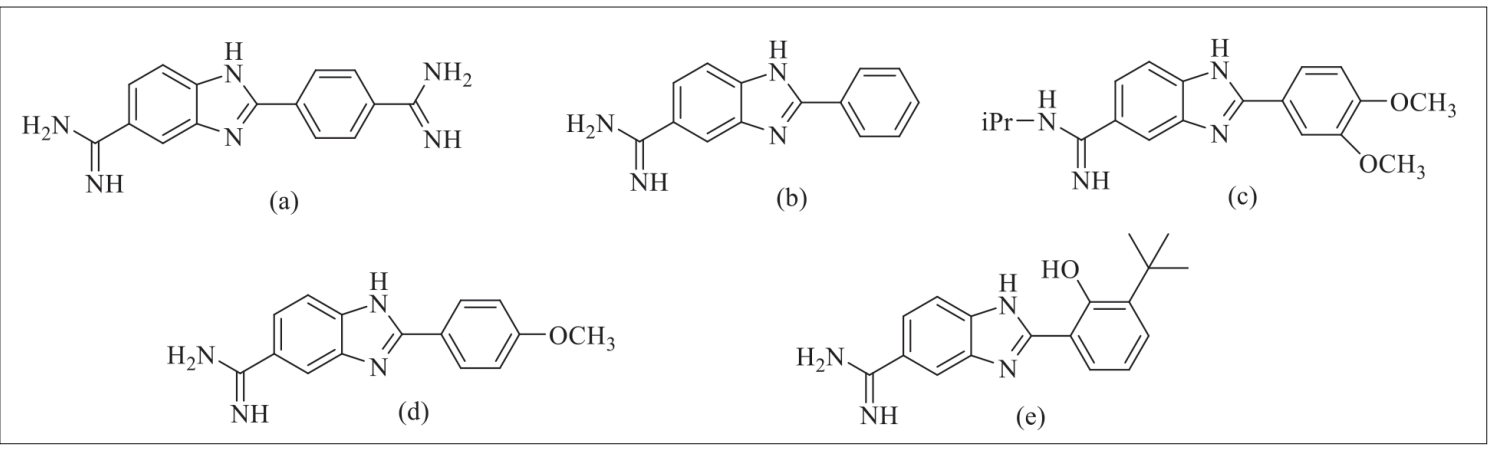

Figure 2. Previously synthesized potent benzimidazole carboxamidines 
carboxamidines, we have planned to prepare prodrug structures of some of our effective amidine derivatives which have been previously reported., 1,16 Furthermore we designed new amidoxime derivatives according to literature including amidine derivatives which have potent activities (Figure $2 \mathrm{~d}$, e) ${ }^{17,18}$ In vivo studies of these newly synthesized benzimidazole amidoximes are planned to test efficacy in an animal model and to determine their pharmacokinetic profiles in further analysis.

\section{MATERIALS AND METHODS}

\section{Experimental}

Uncorrected melting points were detected by a capillary melting point device (Büchi B-540). The ${ }^{1} \mathrm{H},{ }^{13} \mathrm{C}$, COSY (Evaluated as only primary neighbourhood), NOESY, HSQC and HMBC - nuclear magnetic resonance (NMR) spectra were performed by VARIAN (Agilent) MERCURY $400 \mathrm{MHz}$ (Varian, Palo Alto, CA, USA) at a proton resonance frequency of $400 \mathrm{MHz}$ and a carbon resonance frequency of $100 \mathrm{MHz}$. The optimisation of NMR spectrum was directed by Agilent $\mathrm{Vnmr} J$ version 3.2 revision Asoftware. The samples to be analysed (5-20 mg) were prepared in $0.7 \mathrm{ml}$ of $\mathrm{CD}_{3} \mathrm{OD}, \mathrm{CDCl}_{3}$ or dimethyl sulfoxide (DMSO) $-d_{6}$ and tetramethylsilane was used as an internal standard. The liquid chromatography-mass spectrometry (LC-MS) spectra were obtained by using the electrospray-ionization (ESI) (+) method on a Waters Micro mass ZQ connected with Waters Alliance high-performance liquid chromatography (Waters Corporation, Milford, USA), with a C-18 column (X Terra, 4.6 X 250mm, $5 \mu \mathrm{m}$ ). Because of the tautomeric forms of these compounds, ${ }^{1} \mathrm{H}$ and ${ }^{13} \mathrm{C}$-NMR spectra of some unsubstituted analogues could not be clearly seen and appearance of some proton and carbon signals as broad peaks and unobservable some hinge carbon signals are normal. In order to remove the tautomeric effect, some of the benzimidazoles were dissolved in $\mathrm{CDCl}_{3}, \mathrm{CD}_{3} \mathrm{OD}$ or DMSO- $d_{6}$, followed by dry $\mathrm{NaH}$, and $\mathrm{D}_{2} \mathrm{O}$ were added to the NMR tube and stirred well. Besides substitution of this "nitrogen atom's proton" with an alkyl/aryl group has appeared to prevent the tautomerism.

\section{Chemistry}

The synthetic pathways for preparation of targeted compounds are outlined in Scheme1. All commercially available compounds were supplied from Sigma Aldrich. 4-Amino-3-nitrobenzonitrile is a commercially available compound. Compound $1-4^{2,19}$ and compound 5-7,920-22 were prepared according to the given literature methods. Compound 8 was prepared from compound 3 by hydrogenation reaction. Compound 10-19 were obtained by condensation of 3-amino-4-( $\mathrm{N}$-substituteamino)benzonitriles with $\mathrm{Na}_{2} \mathrm{~S}_{2} \mathrm{O}_{5}$ adduct of related arylaldehydes in dimethylformamide (DMF). ${ }^{23}$ Compound 19 was prepared from compound 11 according to the literature. ${ }^{24}$ The nitrile groups of these benzimidazole carbonitriles were converted to $N$-hydroxy benzamidine derivatives (amidoxime derivatives, 20-29) with the presence of $\mathrm{NH}_{2} \mathrm{OH} . \mathrm{HCl}$ and $\mathrm{KOtBu}$ in DMSO. ${ }^{25}$

\section{3-Amino-4- (phenylamino) benzonitrile 8}

Compound 3 ( $2 \mathrm{mmol}$ ) was dissolved in ethanol $(50 \mathrm{~mL})$ and was hydrogenated by $\mathrm{H}_{2}$ (40 psi) and $\mathrm{Pd}-\mathrm{C}(10 \%, 25 \mathrm{mg}$ ) until uptake of $\mathrm{H}_{2}$ ceased. Then the $\mathrm{Pd}-\mathrm{C}$ was filtered off from celite and washed with ethanol several times. The filtrate was concentrated in vacuo and the crude product was used for further steps without crystallization. Yield, 0.67g (92\%). Mp: $152-154^{\circ} \mathrm{C} .{ }^{1} \mathrm{H}-\mathrm{NMR} \delta$ (DMSO-d $)$ : 5.21 (s, $2 \mathrm{H},-\mathrm{NH}_{2}$ ), 6.86-6.90 $(\mathrm{m}, 2 \mathrm{H}), 6.97-7.01(\mathrm{~m}, 3 \mathrm{H}), 7.06(\mathrm{~d}, 1 \mathrm{H}, \mathrm{J}=8 \mathrm{~Hz}), 7.24(\mathrm{t}, 2 \mathrm{H}, \mathrm{J}=$ $7.6 \mathrm{~Hz}), 7.47(\mathrm{~s}, 1 \mathrm{H}),{ }^{13} \mathrm{C}-\mathrm{NMR} \delta$ (DMSO-d $)$ : 102.1, 116.3, 116.8, 118.3, 120.1, 120.8, 121.1, 129.2, 134.2, 139.3, 142.5. MS (ESI+) m/z: $209.2(\mathrm{M}+\mathrm{H}, 100 \%)$.

\section{Sodium metabisulphite adduct of arylaldehyde derivatives}

The corresponding arylaldehydes $(5 \mathrm{mmol}$ ) were dissolved in ethanol $(25 \mathrm{~mL})$ and the solution of sodium metabisulfite $(0.5 \mathrm{~g})$ in water $(5 \mathrm{~mL})$ was added piece by piece. Then reaction was stirred and kept in refrigerator until all precipitation finished and the resulting precipitate was filtered off and dried, and used without purification for further steps.

\section{General synthesis of compounds 10-19}

The mixture of related 3-amino-4- ( $N$-substituted-amino) benzonitriles 5-9 $(1 \mathrm{mmol})$ and related sodium metabisulphite adduct of arylaldehydes $(1 \mathrm{mmol})$ in DMF $(1 \mathrm{~mL})$ were heated at $120^{\circ} \mathrm{C}$, for $3-4 \mathrm{~h}$. At the end of the time the reaction was cooled and dilute $\mathrm{K}_{2} \mathrm{CO}_{3}$ solution were added. The final precipitate was collected by filtration and dried. If the product was not pure, it was purified with crystallization. Compound $10,{ }^{19} 11,{ }^{24} 12,{ }^{26}$ $14,{ }^{27}$ and $16^{24}$ were prepared according to the given literature methods. Compound 19 was prepared from compound 11 $(0.43 \mathrm{mmol})$ with the reaction of 4 -chlorobenzyl chloride (0.6 mmol) and sodium hydride (95\%) $(0.8 \mathrm{mmol})$ in DMF $(1 \mathrm{~mL})$ and isolated as described in the literature. ${ }^{24}$

\section{1-Butyl-2-(3-(tert-butyl)-2-hydroxyphenyl)-1H-benzo[d]} imidazole-5-carbonitrile 13

Prepared from compound $7(0.189 \mathrm{~g})$ and sodium metabisulphite adduct of 2-hydroxy-3-tert-butylbenzaldehyde (0.282 g) as given in general method and the crude product was crystallized from ethanol. Yield, $0.159 \mathrm{~g}$ (46\%). Mp: $184-187^{\circ} \mathrm{C} .{ }^{1} \mathrm{H}-\mathrm{NMR} \delta$ $\left(\mathrm{DMSO}-d_{6}\right): 0.77\left(\mathrm{t}, 3 \mathrm{H}, \mathrm{J}=7.2 \mathrm{~Hz},-\mathrm{CH}_{3}\right), 1.82-1.24\left(\mathrm{~m}, 2 \mathrm{H},-\mathrm{CH}_{2}\right)$, 1.41(s, 9H, $\left.-\mathrm{CH}_{3}\right), 1.66-1.74\left(\mathrm{~m}, 2 \mathrm{H},-\mathrm{CH}_{2}\right), 4.37(\mathrm{t}, 2 \mathrm{H}, \mathrm{J}=7.6 \mathrm{~Hz}$, $\left.-\mathrm{CH}_{2}\right), 6.98\left(\mathrm{t}, 1 \mathrm{H}, \mathrm{J}=8 \mathrm{~Hz}, \mathrm{H}-5^{\prime}\right), 7.41$ (dd, $1 \mathrm{H}, J=8 \& 1.2 \mathrm{~Hz}$, $\left.H-4^{\prime}\right), 7.52\left(d d, 1 H, J=8 \& 1.2 \mathrm{~Hz}, H-6^{\prime}\right), 7.71(d d, 1 H, J=8.4$ \& $1.2 \mathrm{~Hz}, \mathrm{H}-6), 7.91(\mathrm{~d}, 1 \mathrm{H}, \mathrm{J}=8.8 \mathrm{~Hz}, \mathrm{H}-7), 8.27(\mathrm{~d}, 1 \mathrm{H}, \mathrm{J}=0.8$ $\mathrm{Hz}, \mathrm{H}-4), 11.59$ (s, $1 \mathrm{H}, \mathrm{OH})$. NOESY $\delta\left(\mathrm{DMSO}-d_{6}\right)$ : $\left(\mathrm{N}-\mathrm{CH}_{2} / \mathrm{H}-7\right)$, $\left(\mathrm{N}-\mathrm{CH}_{2} / \mathrm{H}-6^{\prime}\right)$. COSY $\delta\left(\mathrm{DMSO}-d_{6}\right):(\mathrm{H}-6 / \mathrm{H}-7),\left(\mathrm{H}-5^{\prime} / \mathrm{H}-6^{\prime}\right)$, $\left(\mathrm{H}-5^{\prime} / \mathrm{H}-4^{\prime}\right) .{ }^{13} \mathrm{C}-\mathrm{NMR} \& \mathrm{HSQC} \delta\left(\mathrm{DMSO}-d_{6}\right): 13.1,19.1,29.3,30.9$, 34.7, 44.7, 104.5, $112.4(\mathrm{CH}-7), 114.5,118.9\left(\mathrm{CH}-5^{\prime}\right), 119.6,123.3$ (CH-4), 126.1(CH-6), $126.7\left(\mathrm{CH}-6{ }^{\prime}\right), 129.0\left(\mathrm{CH}-4^{\prime}\right), 137.6,138.1$, 140.2, 153.9, 155.7. MS (ESI+) m/z: $348.94(\mathrm{M}+\mathrm{H}, 100 \%)$.

\section{1-Butyl-2-(naphthalen-2-yl)-1H-benzo[d]imidazole-5- carbonitrile 15}

Prepared from compound $7(0.189 \mathrm{~g})$ and sodium metabisulphite adduct of 2 -napthaldehyde $(0.260 \mathrm{~g})$ as given in general method and the crude product was crystallized from ethanol. Yield, 0.133 g, (41\%). Mp: $126-129^{\circ} \mathrm{C} .{ }^{1} \mathrm{H}-\mathrm{NMR} \delta$ (DMSO-d ): $0.72(\mathrm{t}, 3 \mathrm{H}$, $\left.\mathrm{J}=7.6 \mathrm{~Hz},-\mathrm{CH}_{3}\right), 1.11-1.16\left(\mathrm{~m}, 2 \mathrm{H},-\mathrm{CH}_{2}\right), 1.63-1.71\left(\mathrm{~m}, 2 \mathrm{H},-\mathrm{CH}_{2}\right)$, 
$4.47\left(\mathrm{t}, 2 \mathrm{H}, \mathrm{J}=7.2 \mathrm{~Hz},-\mathrm{CH}_{2}\right), 7.63-7.69(\mathrm{~m}, 2 \mathrm{H}), 7.73(\mathrm{dd}, 1 \mathrm{H}, \mathrm{J}=$ $8.4 \& 1.2 \mathrm{~Hz}), 7.92-7.96(\mathrm{~m}, 2 \mathrm{H}), 8.05-8.15(\mathrm{~m}, 3 \mathrm{H}), 8.29(\mathrm{~d}, 1 \mathrm{H}, \mathrm{J}=$ $0.8 \mathrm{~Hz}$ ), $8.42(\mathrm{~s}, 1 \mathrm{H}) .{ }^{13} \mathrm{C}-\mathrm{NMR} \delta$ (DMSO-d $): 13.1,19.1,31.0,44.2$, 104.2, 112.5, 119.8, 124.1, 125.7, 125.9, 126.89, 126.96, 127.5, 127.7, 128.5, 129.0, 132.4, 133.2, 138.7, 142.0, 155.5. MS (ESI+) m/z: $326.68(\mathrm{M}+\mathrm{H}, 100 \%)$.

\section{2-(3,4-Dimethoxyphenyl)-1-phenyl-1H-benzo[d]imidazole-5-} carbonitrile 17

Prepared from compound $8(0.209 \mathrm{~g})$ and sodium metabisulphite adduct of 3,4-dimethoxy benzaldehyde $(0.270 \mathrm{~g})$ as given in general method and the crude product was crystallized from ethanol. Yield, $0.220 \mathrm{~g}(62 \%)$. Mp: $175-176^{\circ} \mathrm{C}$. ${ }^{1} \mathrm{H}-\mathrm{NMR} \delta$ $\left(\mathrm{CDCl}_{3}\right): 3.69\left(\mathrm{~s}, 3 \mathrm{H}, \mathrm{OCH}_{3}\right), 3.87\left(\mathrm{~s}, 3 \mathrm{H}, \mathrm{OCH}_{3}\right), 6.77(\mathrm{~d}, 1 \mathrm{H}, \mathrm{J}=$ $\left.8.4 \mathrm{~Hz}, \mathrm{H}-5^{\prime}\right), 7.10$ (dd, $1 \mathrm{H}, \mathrm{J}=8$ \& $\left.2 \mathrm{~Hz}, \mathrm{H}-6^{\prime}\right), 7.13$ (d, 1H, J= $2 \mathrm{~Hz}, \mathrm{H}-4), 7.24$ (d, 1H, J= $8.8 \mathrm{~Hz}, \mathrm{H}-7$ ), 7.33 (dd, $2 \mathrm{H}, \mathrm{J}=8$ \& 2 $\mathrm{Hz}, \mathrm{H}-2$ ", 6"), 7.49 (dd, $1 \mathrm{H}, J=8.8$ \& $1.6 \mathrm{~Hz}, \mathrm{H}-6), 7.53-7.58$ (m, $\left.3 \mathrm{H}, \mathrm{H}-3^{\prime \prime}, 4^{\prime \prime}, 5^{\prime \prime}\right), 8.16\left(\mathrm{~d}, 1 \mathrm{H}, \mathrm{J}=1.6 \mathrm{~Hz}, \mathrm{H}-2^{\prime}\right)$. COSY $\delta\left(\mathrm{CDCl}_{3}\right)$ : $(\mathrm{H}-6 / \mathrm{H}-7),\left(\mathrm{H}-5^{\prime} / \mathrm{H}-6{ }^{\prime}\right),\left(\mathrm{H}-2^{\prime \prime}, 6^{\prime \prime} / \mathrm{H}-3^{\prime \prime}, 5^{\prime \prime}\right) .{ }^{13} \mathrm{C}-\mathrm{NMR} \delta\left(\mathrm{CDCl}_{3}\right)$ : 55.7, 55.9, 106.2, 110.7, 111.3, 112.2, 119.8, 121.1, 122.8, 124.4, 126.5, $127.4,129.3,130.2,136.4,139.9,142.4,148.7,150.7,154.7 . \mathrm{MS}$ (ESI+) m/z: $356(M+H, 100 \%)$.

1-Benzyl-2-(3,4-dimethoxyphenyl)-1H-benzo[d]imidazole-5carbonitrile 18

Prepared from compound $9(0.223 \mathrm{~g})$ and sodium metabisulphite adduct of 3,4-dimethoxy benzaldehyde $(0.270 \mathrm{~g})$ as given in general method the crude product was crystallized from<smiles>[R]Nc1ccc(C#N)cc1N</smiles><smiles>[R]c1[Y](C)c([R3])c([R])c(-c2nc3cc(C#N)ccc3n2[R])c1[R]</smiles>

10-19<smiles>[R3][Y]1c([R3])c([R])c(-c2nc3cc(/C(N)=N\O)ccc3n2[R])c([R])c1[R3]</smiles>

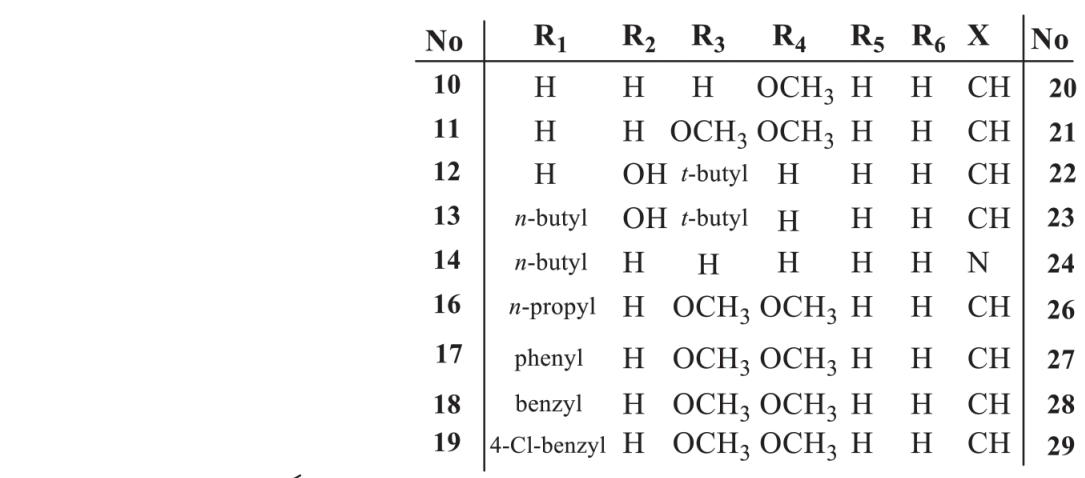

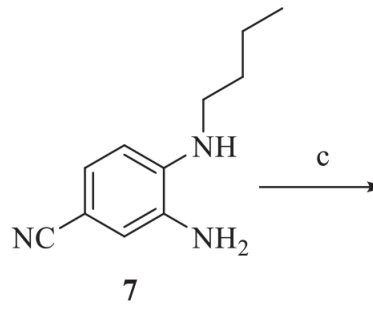

Reagen<smiles>CCCCn1c(-c2ccc3ccccc3c2)nc2cc(C#N)ccc21</smiles>

15

b: $\mathrm{H}_{2} / \mathrm{Pd}-\mathrm{C}$; c:corresponding $\mathrm{Na}_{2} \mathrm{~S}_{2} \mathrm{O}_{5}$ adduct of arylaldehydes in DMF; d: $\mathrm{NH}_{2} \mathrm{OH} . \mathrm{HCl} / \mathrm{KO} . t-\mathrm{Bu}, \mathrm{DMSO}$. 
ethanol. Yield, $0.191 \mathrm{~g}(52 \%)$. Mp: $203-204^{\circ} \mathrm{C}$. ${ }^{1} \mathrm{H}-\mathrm{NMR} \delta\left(\mathrm{CDCl}_{3}\right)$ : 3.73 (s, 3H, OCH $), 3.93$ (s, 3H, OCH $), 5.51$ (s, $2 \mathrm{H},-\mathrm{CH}_{2}$ benzyl), 6.93 (d, $\left.1 \mathrm{H}, \mathrm{J}=8.4 \mathrm{~Hz}, \mathrm{H}-5^{\prime}\right), 7.10$ (dd, $2 \mathrm{H}, \mathrm{J}=8$ \& $1.2 \mathrm{~Hz}, \mathrm{H}-2^{\prime \prime}, 6^{\prime \prime}$ ), 7.22 (dd, $1 \mathrm{H}, J=8.8$ \& $\left.2 \mathrm{~Hz}, \mathrm{H}-6^{\prime}\right), 7.25(\mathrm{~d}, 1 \mathrm{H}, J=2 \mathrm{~Hz}, \mathrm{H}-4)$, 7.29 (d, $1 \mathrm{H}, J=8 \mathrm{~Hz}, \mathrm{H}-7$ ), 7.33-7.40 (m, 3H, H-3",4",5"), 7.49 (dd, $1 \mathrm{H}, J=8.8$ \& $1.6 \mathrm{~Hz}, \mathrm{H}-6), 8.17$ (d, $\left.1 \mathrm{H}, J=0.8 \mathrm{~Hz}, \mathrm{H}-2^{\prime}\right)$. COSY $\delta\left(\mathrm{CDCl}_{3}\right):(\mathrm{H}-6 / \mathrm{H}-7),\left(\mathrm{H}-5^{\prime} / \mathrm{H}-6^{\prime}\right),\left(\mathrm{H}-2^{\prime \prime}, 6^{\prime \prime} / \mathrm{H}-3^{\prime \prime}, 5^{\prime \prime}\right) .{ }^{13} \mathrm{C}-\mathrm{NMR}$ $\delta\left(\mathrm{CDCl}_{3}\right): 48.7,55.7,56.0,105.9,111.1,111.3,112.1,119.8,121.2$, 122.0, 124.6, 125.6, 126.3, 128.2, 129.3, 135.6, 138.9, 144.6, 149.2, 151.1, 156.6. MS (ESI+) m/z: $369(M+H, 100 \%)$.

\section{General synthesis of compounds 20-29}

Benzimidazole carbonitriles $10-19(1 \mathrm{mmol})$ were stirred with a mixture of hydroxylamine hydrochloride $(10 \mathrm{mmol})$ and potassium tert-butoxide $(10 \mathrm{mmol})$ in DMSO $(1 \mathrm{~mL})$ at room temperature for 24h, to furnish the benzimidazole carboxamidoximes 20-29. Then the reaction mixture was cooled and poured into water. The resulting precipitate was collected by filtration and washed with water plenty of time and then dried.

2-(4-Methoxyphenyl)-N-hydroxy-1H-benzo[d]imidazole-5carboximidamide 20

Prepared from compound $10(0.249 \mathrm{~g})$ as given in general method. Yield, 0.231g (82\%). Mp: $268-272^{\circ} \mathrm{C}$. ${ }^{1} \mathrm{H}-\mathrm{NMR} \delta$ (DMSO- $d_{6}$ ): 3.82 (s, $\left.3 \mathrm{H}, \mathrm{OCH}_{3}\right), 5.76\left(\mathrm{~s}, 2 \mathrm{H}\right.$, amidoxime $\left.\mathrm{NH}_{2}\right), 7.09(\mathrm{~d}, 2 \mathrm{H}, \mathrm{J}=8.4$ $\left.\mathrm{Hz}, \mathrm{H}-3^{\prime}, 5^{\prime}\right), 7.46-7.92(\mathrm{~m}, 3 \mathrm{H}), 8.10\left(\mathrm{~d}, 2 \mathrm{H}, \mathrm{J}=8.8 \mathrm{~Hz}, \mathrm{H}-2^{\prime}, 6^{\prime}\right.$ ), 9.49 (s, $1 \mathrm{H}$, amidoxime $\mathrm{OH}$ ), 12.75 (s, $1 \mathrm{H}$, imidazole $\mathrm{NH}$ ). COSY $\delta$ (DMSO- $\left.d_{6}\right):\left(H-2^{\prime}, 6^{\prime} / H-3^{\prime}, 5^{\prime}\right)$. NOESY $\delta\left(\right.$ DMSO- $\left.d_{6}\right):\left(-\mathrm{OCH}_{3} /\right.$ $\left.\mathrm{H}-3^{\prime}, 5^{\prime}\right)\left(\mathrm{H}-2^{\prime}, 6^{\prime} / \mathrm{H}-3^{\prime}, 5^{\prime}\right) .{ }^{13} \mathrm{C}-\mathrm{NMR} \delta$ (DMSO-d $)$ : 55.3, 71.3, 108.2, 114.3, 117.7, 119.7, 120.0, 122.5, 127.5, 128.0, 151.6, 152.1, 160.7. MS (ESI+) m/z: $283.6(\mathrm{M}+\mathrm{H}, 100 \%)$.

2-(3,4-Dimethoxyphenyl)-N-hydroxy-1H-benzo[d]imidazole5-carboximidamide 21

Prepared from compound $11(0.279 \mathrm{~g})$ as given in general method. Yield, $0.237 \mathrm{~g}(76 \%)$ ) Mp: $217-219^{\circ} \mathrm{C} .{ }^{1} \mathrm{H}-\mathrm{NMR} \delta$ (DMSO- $d_{6}$ $\left.+\mathrm{NaH}+\mathrm{D}_{2} \mathrm{O}\right): 3.76\left(\mathrm{~s}, 3 \mathrm{H}, \mathrm{OCH}_{3}\right), 3.83\left(\mathrm{~s}, 3 \mathrm{H}, \mathrm{OCH}_{3}\right), 6.91(\mathrm{~d}, 1 \mathrm{H}$, $\left.J=8.8 \mathrm{~Hz}, \mathrm{H}-5^{\prime}\right), 7.15(\mathrm{dd}, 1 \mathrm{H}, J=8 \& 1.6 \mathrm{~Hz}, \mathrm{H}-6), 7.30(\mathrm{~d}, 1 \mathrm{H}, J=$ $8.8 \mathrm{~Hz}, \mathrm{H}-7), 7.68(\mathrm{~d}, 1 \mathrm{H}, J=1.2 \mathrm{~Hz}, \mathrm{H}-4), 7.80(\mathrm{~d}, 1 \mathrm{H}, J=8 \& 2 \mathrm{~Hz}$, $\left.\mathrm{H}-6^{\prime}\right), 7.93\left(\mathrm{~d}, 1 \mathrm{H}, \mathrm{J}=1.6 \mathrm{~Hz}, \mathrm{H}-2^{\prime}\right)$. COSY $\delta\left(\mathrm{DMSO}-\mathrm{d}_{6}+\mathrm{NaH}+\mathrm{D}_{2} \mathrm{O}\right.$ ): $(\mathrm{H}-6 / \mathrm{H}-7),\left(\mathrm{H}-5^{\prime} / \mathrm{H}-6^{\prime}\right) .{ }^{13} \mathrm{C}-\mathrm{NMR} \delta\left(\mathrm{DMSO}-d_{6}+\mathrm{NaH}+\mathrm{D}_{2} \mathrm{O}\right): 48.7$, 55.7, 55.8, $110.9\left(\mathrm{CH}-2^{\prime}\right), 111.8\left(\mathrm{CH}-5^{\prime}\right), 112.9(\mathrm{CH}-4), 114.9,115.5$, $119.2(\mathrm{CH}-6$ ') $, 123.1,130.8,147.1,148.2,148.5,153.3,161.2$. MS (ESI+) m/z: $313(M+H, 100 \%)$.

\section{2-[3-(tert-Butyl)-2-hydroxyphenyl]-N-hydroxy-1H-benzo[d] imidazole-5-carboximidamide 22}

Prepared from compound $12(0.291 \mathrm{~g})$ as given in general method. Yield, $0.233 \mathrm{~g} 72 \%$. Mp: $199-201^{\circ} \mathrm{C}$. ${ }^{1} \mathrm{H}-\mathrm{NMR} \delta\left(\mathrm{CD}_{3} \mathrm{OD}\right)$ : $1.47(\mathrm{~s}, 9 \mathrm{H}), 6.87(\mathrm{t}, 1 \mathrm{H}, \mathrm{J}=8 \mathrm{~Hz}), 7.36(\mathrm{dd}, 1 \mathrm{H}, J=7.6 \& 1.2 \mathrm{~Hz}$ ), 7.57-7.59 (m, 2H), $7.73(\mathrm{~d}, 1 \mathrm{H}, J=6.8 \mathrm{~Hz}), 7.92-7.94(\mathrm{~m}, 1 \mathrm{H})$. ${ }^{13} \mathrm{C}-\mathrm{NMR} \delta\left(\mathrm{CD}_{3} \mathrm{OD}\right): 30.0,35.9,40.5,110.6,111.9,113.7,117.5$, 118.8, 119.5, 122.9, 124.9, 129.3, 130.0, 139.0, 145.2, 155.4, 156.4, 159.2 MS (ESI+) m/z: $325.43(\mathrm{M}+\mathrm{H}, 100 \%)$.

1-Butyl-2-(3-(tert-butyl)-2-hydroxyphenyl)-N-hydroxy-1Hbenzo[d]imidazole-5-carboximidamide 23

Prepared from compound $13(0.347 \mathrm{~g})$ as given in general method. Yield, $0.296 \mathrm{~g}$ 78\%. Mp: $205-208^{\circ} \mathrm{C}$. ${ }^{1} \mathrm{H}-\mathrm{NMR} \delta$ (DMSO- $d_{6}$ ): $0.85\left(\mathrm{t}, 3 \mathrm{H}, \mathrm{J}=7.2 \mathrm{~Hz},-\mathrm{CH}_{3}\right), 1.25-1.30(\mathrm{~m}, 2 \mathrm{H}$, $\left.-\mathrm{CH}_{2}\right), 1.47\left(\mathrm{~s}, 9 \mathrm{H},-\mathrm{CH}_{3}\right), 1.76-1.82\left(\mathrm{~m}, 2 \mathrm{H},-\mathrm{CH}_{2}\right), 3.37(\mathrm{t}, 2 \mathrm{H}, \mathrm{J}=$ $\left.7.6 \mathrm{~Hz}, \mathrm{H}-5^{\prime}\right), 7.43-7.48\left(\mathrm{~m}, 2 \mathrm{H}, \mathrm{H}-4^{\prime}, 6^{\prime}\right), 7.58(\mathrm{~d}, 1 \mathrm{H}, \mathrm{J}=8.8 \mathrm{~Hz}$, $\mathrm{H}-7$ ), 7.67 (dd, $1 \mathrm{H}, J=8.4 \& 1.6 \mathrm{~Hz}, \mathrm{H}-6), 7.99(\mathrm{~d}, 1 \mathrm{H}, J=1.2 \mathrm{~Hz}$, $\mathrm{H}-4)$. COSY $\delta$ (DMSO- $\left.d_{6}\right):(\mathrm{H}-6 / \mathrm{H}-7),\left(\mathrm{H}-5^{\prime} / \mathrm{H}-4^{\prime}\right),\left(\mathrm{H}-5^{\prime} / \mathrm{H}-6^{\prime}\right)$. NOESY $\delta$ (DMSO- $\left.d_{6}\right):\left(-\mathrm{N}-\mathrm{CH}_{2} / \mathrm{H}-7\right),\left(-\mathrm{N}-\mathrm{CH}_{2} / \mathrm{H}-6^{\prime}\right) .{ }^{13} \mathrm{C}-\mathrm{NMR}$ $\delta$ (DMSO- $d_{6}$ ): 13.8, 20.8, 30.1, 32.7, 36.1, 46.2, 111.7, 116.3, 117.8, 120.0, 122.9, 127.7, 129.2, 130.1, 137.7, 139.6, 142.5, 154.5, 157.4. MS (ESI+) m/z: $381.83(\mathrm{M}+\mathrm{H}, 100 \%)$.

1-Butyl-N-hydroxy-2-(pyridin-4-yl)-1H-benzo[d]imidazole-5carboximidamide 24

Prepared from compound $14(0.276 \mathrm{~g})$ as given in general method. Yield, $0.200 \mathrm{~g}(65 \%)$. Mp: $232-235^{\circ} \mathrm{C}$. ${ }^{1} \mathrm{H}-\mathrm{NMR} \delta$ (DMSO- $d_{6}$ ): 0.76 $\left(\mathrm{t}, 3 \mathrm{H}, \mathrm{J}=7.2 \mathrm{~Hz},-\mathrm{CH}_{3}\right), 1.12-1.18\left(\mathrm{~m}, 2 \mathrm{H},-\mathrm{CH}_{2}\right), 1.64-1.68(\mathrm{~m}, 2 \mathrm{H}$, $\left.-\mathrm{CH}_{2}\right), 4.38\left(\mathrm{t}, 2 \mathrm{H}, \mathrm{J}=6.8 \mathrm{~Hz}, \mathrm{~N}-\mathrm{CH}_{2}\right), 5.87(\mathrm{~s}, 2 \mathrm{H}$, amidoxime $\mathrm{NH}_{2}$ ), $7.68(\mathrm{~d}, 1 \mathrm{H}, J=8.8 \mathrm{~Hz}, \mathrm{H}-7$ ), 7.74 (dd, $1 \mathrm{H}, J=8.8 \& 1.6 \mathrm{~Hz}$, $\mathrm{H}-6$ ), 7.82 (dd, $\left.2 \mathrm{H}, J=4.8 \& 1.6 \mathrm{~Hz}, \mathrm{H}-2^{\prime}, 6^{\prime}\right), 8.04$ (s, 1H, H-4), 8.80 (dd, $\left.2 \mathrm{H}, J=4.8 \& 1.6 \mathrm{~Hz}, \mathrm{H}-3^{\prime}, 5^{\prime}\right), 9.59$ (s, $1 \mathrm{H}$, amidoxime $\mathrm{OH}$ ). COSY $\delta$ (DMSO-d $\mathrm{d}_{6}$ : $(\mathrm{H}-6 / \mathrm{H}-7),\left(\mathrm{H}-2^{\prime}, 6^{\prime} / \mathrm{H}-3^{\prime}, 5^{\prime}\right)$. NOESY $\delta$ (DMSO- $\left.d_{6}\right):\left(\mathrm{N}-\mathrm{CH} 2 / \mathrm{H}-2^{\prime}, 6^{\prime}\right),\left(\mathrm{N}-\mathrm{CH}_{2} / \mathrm{H}-7\right)$, (amidoxime $\mathrm{NH}_{2} /$ $\mathrm{H}-4)$, (amidoxime $\mathrm{OH} / \mathrm{H}-6)$. ROESY $\delta\left(\mathrm{DMSO}-d_{6}\right):\left(\mathrm{N}-\mathrm{CH}_{2} /\right.$ $\left.\mathrm{H}-2^{\prime}, 6^{\prime}\right),\left(\mathrm{N}-\mathrm{CH}_{2} / \mathrm{H}-7\right)$, (amidoxime $\left.\mathrm{NH} 2 / \mathrm{H}-4\right) .{ }^{13} \mathrm{C}-\mathrm{NMR}$ \& HSQC \& HMBC $\delta$ (DMSO-d $\left.d_{6}\right): 13.2,19.1,31.2,43.9,110.6(\mathrm{CH}-7)$, $116.5(\mathrm{CH}-4), 120.9(\mathrm{CH}-6), 123.2\left(\mathrm{CH}-2^{\prime}, 6^{\prime}\right), 127.9(\mathrm{C}-5), 136.3$ (amidoxime C), 137.7 (C-1'), 142.2 (C-3a), $150.2\left(\mathrm{CH}-3^{\prime}, 5^{\prime}\right), 150.8$ (C-2), 151.2 (C-7a). MS (ESI+) m/z: $310.48(\mathrm{M}+\mathrm{H}, 100 \%)$.

1-Butyl-N-hydroxy-2-(naphthalen-2-yl)-1H-benzo[d] imidazole-5-carboximidamide 25

Prepared from compound $15(0.325 \mathrm{~g})$ as given in general method. Yield, $0.243 \mathrm{~g}(68 \%)$. Mp: $231-233^{\circ} \mathrm{C}$. ${ }^{1} \mathrm{H}-\mathrm{NMR} \delta$ $\left(\mathrm{CD}_{3} \mathrm{OD}\right): 0.72\left(\mathrm{t}, 3 \mathrm{H}, \mathrm{J}=7.6 \mathrm{~Hz},-\mathrm{CH}_{3}\right), 1.10-1.16\left(\mathrm{~m}, 2 \mathrm{H},-\mathrm{CH}_{2}\right.$ ), $1.67-1.70\left(\mathrm{~m}, 2 \mathrm{H},-\mathrm{CH}_{2}\right), 4.42\left(\mathrm{t}, 2 \mathrm{H}, \mathrm{J}=7.2 \mathrm{~Hz},-\mathrm{CH}_{2}\right), 5.89(\mathrm{~s}$, $2 \mathrm{H}$, amidoxime $\left.\mathrm{NH}_{2}\right), 7.63-7.69(\mathrm{~m}, 4 \mathrm{H}), 7.92(\mathrm{dd}, 1 \mathrm{H}, \mathrm{J}=8.4 \& 1.2$ $\mathrm{Hz}), 8.03-8.13(\mathrm{~m}, 4 \mathrm{H}), 8.38(\mathrm{~s}, 1 \mathrm{H}), 9.61(\mathrm{~s}, 1 \mathrm{H}$, amidoxime $\mathrm{OH})$. ${ }^{13} \mathrm{C}-\mathrm{NMR} \delta\left(\mathrm{CD}_{3} \mathrm{OD}\right): 13.2,19.1,31.2,43.9,110.5,116.3,120.4,126.3$, 127.3, 127.6, 127.7, 127.8, 128.3, 128.5, 128.7, 132.5, 133.1, 136.3, 142.4, 151.4, 153.6. MS (ESI+) m/z: $359.8(\mathrm{M}+\mathrm{H}, 100 \%)$.

\section{2-(3,4-Dimethoxyphenyl)-N-hydroxy-1-propyl-1H-benzo[d]} imidazole-5-carboximidamide 26

Prepared from compound $16(0.321 \mathrm{~g})$ as given in general method. Yield, 0.290 g $82 \%$. Mp: $245-247^{\circ} \mathrm{C}$. ${ }^{1} \mathrm{H}-\mathrm{NMR} \delta$ (DMSO-d $_{6}$ ): 0.76 $\left(\mathrm{t}, 3 \mathrm{H}, \mathrm{J}=7.2 \mathrm{~Hz},-\mathrm{CH}_{3}\right), 1.71\left(\mathrm{~m}, 2 \mathrm{H},-\mathrm{CH}_{2\left(2^{\prime \prime}\right)}\right), 3.84\left(\mathrm{~s}, 3 \mathrm{H}, \mathrm{OCH}_{3}\right.$ ), $3.85\left(\mathrm{~s}, 3 \mathrm{H}, \mathrm{OCH}_{3}\right), 4.27\left(\mathrm{t}, 2 \mathrm{H}, \mathrm{J}=7.2 \mathrm{~Hz},-\mathrm{CH}_{2\left(\mathrm{I}^{\prime}\right)}\right), 5.83(\mathrm{~s}, 2 \mathrm{H}$, amidine $\mathrm{NH}$ ), $7.14\left(\mathrm{~d}, 1 \mathrm{H}, \mathrm{J}=8.4 \mathrm{~Hz}, \mathrm{H}-5^{\prime}\right), 7.30-7.33(\mathrm{~m}, 2 \mathrm{H}, \mathrm{H}-4$, 6'), 7.60-7.66 (m, 2H, H-6,7), $7.97\left(\mathrm{~d}, 1 \mathrm{H}, J=0.8 \mathrm{~Hz}, \mathrm{H}-2^{\prime}\right), 9.55$ (s, $1 \mathrm{H}$, amidoxime $\mathrm{OH}$ ). COSY $\delta$ (DMSO- $\left.d_{6}\right):(\mathrm{H}-6 / \mathrm{H}-7),\left(\mathrm{H}-5^{\prime} / \mathrm{H}-6^{\prime}\right)$. ${ }^{13} \mathrm{C}-\mathrm{NMR} \delta\left(\mathrm{DMSO}-d_{6}\right): 10.9\left(\mathrm{CH}_{3}\right), 22.6\left(\mathrm{CH}_{2\left(2^{\prime \prime}\right)}\right), 45.8\left(\mathrm{CH}_{2\left(1^{\prime \prime}\right)}\right)$, $55.5\left(\mathrm{OCH}_{3}\right), 55.6\left(\mathrm{OCH}_{3}\right), 110.3(\mathrm{CH}-7), 111.6\left(\mathrm{CH}-5^{\prime}\right), 112.5(\mathrm{CH}-$ 4), $115.9\left(\mathrm{CH}-2^{\prime}\right), 120.1(\mathrm{CH}-6), 121.7\left(\mathrm{CH}-6^{\prime}\right), 122.7,127.4,136.3$, 142.2, 148.6, 149.9, 151.4, 153.7. MS (ESI+) m/z: $355(M+H, 100 \%)$.

2-(3,4-Dimethoxyphenyl)-N-hydroxy-1-phenyl-1H-benzo[d] imidazole-5-carboximidamide 27 
Prepared from compound $17(0.355 \mathrm{~g})$ as given in general method. Yield, $0.295 \mathrm{~g}$ (76\%). Mp: $244-246^{\circ} \mathrm{C} .{ }^{1} \mathrm{H}-\mathrm{NMR} \delta\left(\mathrm{CD}_{3} \mathrm{OD}\right)$ : 3.62 (s, 3H, OCH $), 3.83$ (s, 3H, OCH $\mathrm{H}_{3}$ ), 6.93 (d, 1H, J=8 Hz, H-5'), 7.08 (d, 1H, J=2 Hz, H-2'), 7.16 (dd, 1H, J= 8.8 \& $2 \mathrm{~Hz}, \mathrm{H}-6^{\prime}$ ), $7.23(\mathrm{~d}, 1 \mathrm{H}, \mathrm{J}=8.4 \mathrm{~Hz}, \mathrm{H}-7), 7.39-7.42\left(\mathrm{~m}, 2 \mathrm{H}, \mathrm{H}-2^{\prime \prime}, 6\right.$ 6") $^{\prime}, 7.56-$ $7.62\left(\mathrm{~m}, 4 \mathrm{H}, \mathrm{H}-6,3^{\prime \prime}, 4^{\prime \prime}, 5^{\prime \prime}\right), 8.04(\mathrm{~d}, 1 \mathrm{H}, \mathrm{J}=1.6 \mathrm{~Hz}, \mathrm{H}-4)$ ). COSY $\delta$ (CD $\mathrm{OD}):(\mathrm{H}-6 / \mathrm{H}-7),\left(\mathrm{H}-5^{\prime} / \mathrm{H}-6{ }^{\prime}\right),\left(\mathrm{H}-2^{\prime \prime}, 6^{\prime \prime} / \mathrm{H}-3^{\prime \prime}, 5^{\prime \prime}\right) .{ }^{13} \mathrm{C}-\mathrm{NMR} \delta$ (DMSO- $\left.d_{6}+\mathrm{NaH}+\mathrm{D}_{2} \mathrm{O}\right): 55.1,55.5,109.7,111.3,112.3,116.0,120.9$, 121.7, 122.0, 127.6, 128.2, 128.9, 130.1, 136.6, 137.5, 142.2, 148.0, 149.9, 151.2, 152.3. MS (ESI+) m/z: $389(\mathrm{M}+\mathrm{H}, 100 \%)$.

1-Benzyl-2-(3,4-dimethoxyphenyl)-N-hydroxy-1-propyl-1Hbenzo[d]imidazole-5-carboximidamide 28

Prepared from compound $18(0.369 \mathrm{~g})$ as given in general method. Yield, $0.289 \mathrm{~g}$ (72\%). Mp: $224-226^{\circ} \mathrm{C}$. ${ }^{1} \mathrm{H}-\mathrm{NMR} \delta$ (DMSO$\left.d_{6}+\mathrm{NaH}+\mathrm{D}_{2} \mathrm{O}\right): 3.68\left(\mathrm{~s}, 3 \mathrm{H}, \mathrm{OCH}_{3}\right), 3.83\left(\mathrm{~s}, 3 \mathrm{H}, \mathrm{OCH}_{3}\right), 5.59$ (s, $2 \mathrm{H}$, $-\mathrm{CH}_{2}$ benzyl), 7.03 (d, $\left.2 \mathrm{H}, \mathrm{J}=6.8 \mathrm{~Hz}, \mathrm{H}-2^{\prime \prime}, 6^{\prime \prime}\right), 7.10$ (d, $1 \mathrm{H}, \mathrm{J}=8.4$ Hz, H-5'), 7.25-7.34 (m, 5H, H-2',6',3", 4", 5"'), 7.43 (d, 1H, J= 8.8 $\mathrm{Hz}, \mathrm{H}-7$ ), 7.59 (dd, 1H, J= 8.8 \& $1.2 \mathrm{~Hz}, \mathrm{H}-6$ ), 8.02 (s, 1H, H-4). COSY $\delta\left(\mathrm{CDCl}_{3}\right):(\mathrm{H}-6 / \mathrm{H}-7),\left(\mathrm{H}-5^{\prime} / \mathrm{H}-6^{\prime}\right),\left(\mathrm{H}-2^{\prime \prime}, 6^{\prime \prime} / \mathrm{H}-3^{\prime \prime}, 5^{\prime \prime}\right)$. ${ }^{13} \mathrm{C}-\mathrm{NMR} \delta\left(\mathrm{CDCl}_{3}\right): 47.5,55.3,55.5,110.2,111.7,112.4,116.1,120.3$, 121.6, 122.1, 125.8, 127.3, 127.7, 128.7, 136.5, 136.9, 142.3, 148.6, 150.1, 151.3, 153.8. MS (ESI+) m/z: $403(\mathrm{M}+\mathrm{H}, 100 \%)$.

1-(4-Chlorobenzyl)-2-(3,4-dimethoxyphenyl)-N-hydroxy-1Hbenzo[d]imidazole-5-carboximidamide 29

Prepared from compound $19(0.403 \mathrm{~g})$ as given in general method. Yield, $0.305 \mathrm{~g}$ (70\%). Mp: $245-247^{\circ} \mathrm{C}$. ${ }^{1} \mathrm{H}-\mathrm{NMR} \delta$ (DMSO-d ): 3.70 (s, 3H, OCH $), 3.81$ (s, 3H, OCH $\mathrm{H}_{3}$ ), 5.59 (s, $2 \mathrm{H}$, $-\mathrm{CH}_{2}$ benzyl), 5.80 (s, 2H, amidoxime $\mathrm{NH} 2$ ), 7.04 (d, 2H, J= 8.4 $\left.\mathrm{Hz}, \mathrm{H}-2^{\prime \prime}, 6^{\prime \prime}\right), 7.08$ (d, $\left.1 \mathrm{H}, J=8.8 \mathrm{~Hz}, \mathrm{H}-5^{\prime}\right), 7.22-7.24(\mathrm{~m}, 2 \mathrm{H}$, $\left.\mathrm{H}-2^{\prime}, 6^{\prime}\right), 7.37$ (d, 2H, J=8 Hz, H-3", 5'), 7.43 (d, 1H, J= $8.8 \mathrm{~Hz}$, $\mathrm{H}-7), 7.60(\mathrm{~d}, 1 \mathrm{H}, \mathrm{J}=8.8 \mathrm{~Hz}, \mathrm{H}-6), 8.01$ (s, $1 \mathrm{H}, \mathrm{H}-4)$. COSY $\delta$ (DMSO-d ): (H- 6/H-7), (H-5'/H-6'), (H-2",6"/H-3",5"). NOESY $\delta\left(\mathrm{DMSO}_{-} \mathrm{d}_{6}\right):\left(\mathrm{CH}_{2}\right.$ benzyl / H-2',6'), $\left(\mathrm{CH}_{2}\right.$ benzyl / $\left.\mathrm{H}-2^{\prime \prime}, 6^{\prime \prime}\right)$, (amidoxime $\mathrm{NH}_{2} / \mathrm{H}-4$ ), (amidoxime $\mathrm{NH}_{2} / \mathrm{H}-6$ ). ${ }^{13} \mathrm{C}-\mathrm{NMR}$ \& HSQC \& HMBC $\delta\left(\right.$ DMSO- $\left._{6}\right): 46.9$ (benzyl $\left.\mathrm{CH}_{2}\right), 55.4\left(\mathrm{OCH}_{3}\right), 55.6$ $\left(\mathrm{OCH}_{3}\right), 110.2(\mathrm{CH}-7), 111.7\left(\mathrm{CH}-5^{\prime}\right), 112.4\left(\mathrm{CH}-2^{\prime}\right), 116.2(\mathrm{CH}-4)$, 120.5 (CH-6), $121.6\left(\mathrm{CH}-6{ }^{\prime}\right), 121.9$ (C-3'), $127.8\left(\mathrm{CH}-2^{\prime \prime}, 6^{\prime \prime}\right), 128.7$ (CH-3",5"), 131.9, (C-4"), 136.0 (C-1"), 136.4 (C-7a), 142.3 (C-3a), 148.6 (C-1'), 150.2 (C-4'), 151.4 (amidoxime C), 153.9 (C-2). MS (ESI+) m/z: $437.77(\mathrm{M}+\mathrm{H}, 100 \%)$.

\section{RESULTS AND DISCUSSION}

As shown in Scheme 1, uncommercial starting materials, 4(N-substituted-amino) -3-nitrobenzonitriles 1-4, were prepared by nucleophilic displacement of the chloro group of 4-chloro3-nitrobenzonitril with corresponding amine derivatives in N,N-dimethylformamide. Non-susbstituted-4-amino-3nitrobenzonitril is a commercially available compound. Then $\mathrm{Pd} / \mathrm{C}$-catalyzed hydrogenation of these compounds gave $\mathrm{N}$-substituted-3,4-diamino benzonitriles 5-9. The benzimidazole carbonitriles 10-18 were obtained by condensation of these $N$-substituted-3,4-diamino benzonitriles with sodium metabisulfite adduct of related arylaldehydes. Compound 19 was prepared from compound 11 by the alkylation of tautomeric hydrogen with 4-chlorobenzyl chloride with the presense of sodium hydride (95\%) in DMF. Finally targeted $N$-hydroxy benzamidine derivatives 20-29 (amidoxime derivatives) were achieved by the reaction of benzimidazole carbonitriles with $\mathrm{NH}_{2} \mathrm{OH} . \mathrm{HCl}$ and $\mathrm{KOtBu}$ in DMSO. The structures of novel compounds were determined by ${ }^{1} \mathrm{H}-\mathrm{NMR},{ }^{13} \mathrm{C}-\mathrm{NMR}$, some 2D-NMR techniques (COSY, NOESY, HSQC and HMBC) and LC-MS. Benzimidazoles are condensed systems of imidazole and benzene ring, and their hydrogen bearing nitrogen atom resembles the pyrole $N$-atom and the other nitrogen atom resembles the pyridine $\mathrm{N}$-atom. Hydrogen atom of this pyrole $\mathrm{N}$-atom can easily tautomerise in the 1,3-position and because of these tautomeric forms, ${ }^{1} \mathrm{H}$ and ${ }^{13} \mathrm{C}-\mathrm{NMR}$ spectra of unsubstituted compounds may not be clearly seen. Both appearence of some proton and carbon signals as broad peaks and unobservable some hinge carbon signals are normal in that case. Substitution of this $\mathrm{NH}$ proton with an alkyl group would prevent tautomerism and can lead to clearly seen spectra. In this study we can easily see the hydrogen signals of even amidoxime $\mathrm{NH}_{2}$ and $\mathrm{OH}$ in $\mathrm{N}$-alkylated benzimidazoles.

In this study, 10 new amidoxime compounds designed as prodrugs of effective amidine derivatives, were synthesized and their structures were elucidated with advanced NMR techniques.

\section{CONCLUSION}

As a result in this study, a new series of benzimidazole amidoximes 20-29, were synthesized starting from 3-amino-4- (substitutedamino) benzonitrile derivatives and sodium bisulfite adduct of corresponding arylaldehydes. The structures of novel compounds were determined by ${ }^{1} \mathrm{H}-\mathrm{NMR},{ }^{13} \mathrm{C}-\mathrm{NMR}$, some $2 \mathrm{D}-\mathrm{NMR}$ techniques and LC-MS. In our previous studies $1,3,16$ we reported several types of amidino benzimidazoles with their potent antiparasitic, anticancer and antimicrobial activities; however most of the compounds' pharmocokinetic properties and in vivo studies have not been investigated yet. Because of the amidine groups, it seems very likely to have problems in their pharmacokinetic properties, especially in terms of bioavailability. In order to solve this potential problem, in this study we have prepared amidoxime derivatives of these potent amidino benzimidazoles as their prodrugs. In vivo studies of both previous amidine derivatives and amidoxime prodrugs which have been synthesized in this study, are under progress in our ongoing studies.

\section{ACKNOWLEDGEMENTS}

Ankara University Faculty of Pharmacy, Central Laboratory provided support for NMR and Mass spectrometer analysis.

Conflict of Interest: No conflict of interest was declared by the authors.

\section{REFERENCE}

1. Karaaslan C, Kaiser M, Brun R, Göker H. Synthesis and potent antiprotozoal activity of mono/di amidino 2-anilinobenzimidazoles versus Plasmodium falciparum and Trypanosoma brucei rhodesiense. Bioorg Med Chem. 2016;24:4038-4044. 
2. Göker H, Alp M, Yıldız S. Synthesis and Potent Antimicrobial Activity of Some Novel N-(Alkyl)-2-Phenyl-1H-Benzimidazole-5-Carboxamidines. Molecules. 2005;10:1377-1386.

3. Karaaslan C, Bakar F, Goker H. Antiproliferative activity of synthesized some new benzimidazole carboxamidines against MCF-7 breast carcinoma cells. Z Naturforsch C J Biosci. 2018;73:137-145.

4. Ismail MA, Batista-Parra A, Miao Y, Wilson WD, Wenzler T, Brun R, Boykin DW. Dicationic near-linear biphenyl benzimidazole derivatives as DNAtargeted antiprotozoal agents. Bioorg Med Chem. 2005;13:6718-6726.

5. Baraldi PG, Bovero A, Fruttarolo F, Preti D, Tabrizi MA, Pavani MG, Romagnoli R. DNA minor groove binders as potential antitumor and antimicrobial agents. Med Res Rev. 2004;24:475-528.

6. Hranjec M, Piantanida I, Kralj M, Suman L, Pavelic K, Karminski- Zamola G. Novel amidino-substituted thienyl- and furylvinylbenzimidazole: derivatives and their photochemical conversion into corresponding diazacyclopenta[c]fluorenes. Synthesis, interactions with DNA and RNA, and antitumor evaluation. J Med Chem. 2008;51:4899-4910.

7. Starcevic K, Kralj M, Ester K, Sabol I, Grce M, Pavelic K, KarminskiZamola G. Synthesis, antiviral and antitumor activity of 2-substituted5-amidino-benzimidazoles. Bioorg Med Chem. 2007;15:4419-4426.

8. Hranjec M, Starcevic K, Piantanida I, Kralj M, Marjanovic M, Hasani M, Westman G, Karminski-Zamola G. Synthesis, antitumor evaluation and DNAbinding studies of novel amidino-benzimidazolyl substituted derivatives of furyl-phenyl- and thienyl-phenyl-acrylates, naphthofurans and naphthothiophenes. Eur J Med Chem. 2008;43:2877-2890.

9. Qiu G, Jiang J, Liu XS. Pentamidine sensitizes chronic myelogenous leukemia K562 cells to trail-induced apoptosis. Leuk Res. 2012;36:14171421.

10. Pathak MK, Dhawan D, Lindner DJ, Borden EC, Farver C, Yi T. Pentamidine is an inhibitor of $P R L$ phosphatases with anticancer activity. Mol Cancer Ther. 2002;1:1255-1264.

11. Jung HJ, Suh SI, Suh MH, Baek WK, Park JW. Pentamidine reduces expression of hypoxia-inducible factor-1 $\alpha$ in DU145 and MDAMB- 231 cancer cells. Cancer Lett. 2011;303:39-46.

12. Reeh C, Wundt J, Clement B. N,N'-Dihydroxyamidines: A New Prodrug Principle To Improve the Oral Bioavailability of Amidines. J Med Chem. 2007;50:6730-6734.

13. Berger O, Ortial S, Wein S, Denoyelle S, Bressolle F, Durand T, Escale $\mathrm{R}$, Vial H J, Vo-Hoang Y. Evaluation of amidoxime derivatives as prodrug candidates of potent biscationic antimalarials. Bioorg Med Chem Lett. 2019;29:2203-2207.

14. Boykin DW. Antimicrobial activity of the DNA minor groove binders furamidine and analogs. J Braz Chem Soc. 2002;13:763.
15. Clement B. Pharmaceutical preparations that contain an active substance with modified amidine groups. Patents DE 4321444, WO 9501168, EP 0708640, US 5786383, and JP 3838657, 1995.

16. Karaaslan C, Duydu Y, Ustundag A, Yalcin CO, Kaskatepe B, Goker H. Synthesis \& Anticancer Evaluation of New Substituted 2-(3,4-Dimethoxyphenyl) benzazoles. Med Chem. 2019;15:287-297.

17. Chavda S, Dittenhafer K, Wu K, Merrick C, Desta D, Cordes E, Babu $B$, Tzou S, Brockway O, Sjoholm R, Lee M. DNA sequence-selective monoheterocyclic analog of Hoechst 33258: cytotoxicity and antiparasitic properties. Heterocycl Commun. 2010;16:227-230.

18. Weidner-Wells MA, Ohemeng KA, Nguyen VN, Fraga-Spano S, Macielag MJ, Werblood HM, Foleno BD, Webb GC, Barrett JF, Hlasta DJ. Amidino Benzimidazole Inhibitors of Bacterial Two-Component Systems. Bioorg Med Chem Lett. 2001;11:1545-1548.

19. Goker H, Kus C, Boykin DW, Yildiz S, Altanlar N. Synthesis of Some New 2 Substituted-phenyl-1H-benzimidazole-5-carbonitriles and Their Potent Activity Against Candida Species. Bioorg Med Chem. 2002;10:25892596.

20. Fairley TA, Tidvell RR, Donkor I, Naiman NA, Ohemeng KA, Lombardy RJ, Bentley JA, Cory M. Structure, DNA Minor Groove Binding, and Base Pair Specificity of Alkyl- and Aryl-Linked Bis (amidinobenzimidazoles) and Bis (amidinoindoles). J Med Chem. 1993;36:1746-1753.

21. Goker H, Tuncbilek M, Suzen S, Kus C, Altanlar N. Synthesis and Antimicrobial Activity of Some New 2-Phenyl-N-substituted Carboxamido-1H-benzimidazole Derivatives, Arch Pharm Pharm Med Chem. 2001;334:148-152.

22. Goker H, Boykin DW. Ring Cleavage of bis-cyanosubstituted benzimidazoles by DIBAL. Heterocyc Comm. 2001;7:6,529-534.

23. Ridley HF, Spickett RGW, Timmis GM. A new synthesis of benzimidazoles and aza-analogs. J Heterocycl Chem. 1965;2:453-456.

24. Doganc F, Alp M, Goker H. Separation and identification of the mixture of 2-(3,4-dimethoxyphenyl)-1-n-propyl or (4-chlorobenzyl)-5 and (6)-1H-benzimidazole carbonitriles. Magn Reson Chem. 2016;54:851857.

25. Farahat AA, Bennett-Vaughn C, Mineva EM, Kumar A, Wenzler T, Brun R, Liu Y, Wilson WD, Boykin DW. Synthesis, DNA binding and antitrypanosomal activity of benzimidazole analogues of DAPI. Bioorg Med Chem Lett. 2016;26:5907-5910.

26. Puskullu MO, Yıldız S, Goker H. Synthesis and Antistaphylococcal Activity of $\mathrm{N}$-Substituted-1H-benzimidazole-sulphonamides. Arch Pharm Chem Life Sci. 2010;343:31-39.

27. Goker H, Alp M, Ates-Alagoz Z, Yıldız S. Synthesis and Potent Antifungal Activity Against Candida Species of Some Novel 1H-Benzimidazoles. J Het Chem. 2009;46:936-948. 